Théologiques

Théologiques

\title{
La réappropriation de la dimension spirituelle en sciences infirmières
}

\section{Jacinthe Pepin}

Volume 9, numéro 2, automne 2001

Sens et spiritualité dans les pratiques professionnelles

URI : https://id.erudit.org/iderudit/007294ar

DOI : https://doi.org/10.7202/007294ar

Aller au sommaire du numéro

Éditeur(s)

Faculté de théologie de l'Université de Montréal

ISSN

1188-7109 (imprimé)

1492-1413 (numérique)

Découvrir la revue

Citer cet article

Pepin, J. (2001). La réappropriation de la dimension spirituelle en sciences infirmières. Théologiques, 9(2), 33-46. https://doi.org/10.7202/007294ar d'utilisation que vous pouvez consulter en ligne.

https://apropos.erudit.org/fr/usagers/politique-dutilisation/ 


\title{
La réappropriation de la dimension spirituelle en sciences infirmières
}

\author{
Jacinthe PEPIN, inf. Ph.D \\ Chantal CARA, inf. Ph.D \\ Faculté des sciences infirmières \\ Université de Montréal
}

Dans cet article, nous soutiendrons que les discours émergents sur le sens, la spiritualité et les religions dans le domaine de la santé, et particulièrement dans le milieu infirmier, sont le signe d'une re-connaissance ou encore d'une re-découverte de la dimension spirituelle de l'humain, de même que du lien entre cette dimension spirituelle et la santé. C'est essentiellement à travers le paradigme holiste ou unitaire ${ }^{1}$, à travers la vision de l'humain comme étant indivisible, «corps-âme-esprit $»^{2}$, que des questionnements sur nos façons de comprendre la personne, la santé, la vie / la mort, et le soin surviennent; que la dimension spirituelle de l'humain resurgit. Ces questionnements semblent rampants, rejoignant les professionnels de la santé autant dans leur vie personnelle que professionnelle; dans leur pratique, leur enseignement et leur recherche.

Étant infirmières, nous nous attarderons particulièrement à l'émergence des discours sur le sens et la spiritualité en sciences infirmières dans les trois champs suivants : la pratique professionnelle, les écrits conceptuels et théoriques de même que les écrits empiriques.

Mais, tout d'abord, qu'entendons-nous par spiritualité dans le domaine des sciences infirmières ? Pour certains, ce terme correspond à la religion de la personne soignée. Toutefois, les écrits scientifiques

1. M.A Newman, A.M. Sime et S.A. Corcoran-Perry, "The Focus of the Discipline of Nursing ", Advances in Nursing Science, 14/1 (1991) p. 1-6. 2. J. Watson, Nursing. The Philosophy and Science of Caring, Boston, Little Brown, 1979. 
de la discipline infirmière insistent de plus en plus sur une vision élargie de ce concept et on y trouve principalement quatre types de définitions. Par exemple, des auteurs définissent la spiritualité comme étant une relation avec soi-même, les autres, l'environnement et ce qui est plus grand que soi (Dieu) ${ }^{3}$. Cette définition, de nature relationnelle, fait appel à un sens profond d'être lié à ce qui est. Pour Macrae et Watson, la spiritualité correspond plutôt à l'essence de la personne, laquelle demeure intrinsèque à l'être humain ${ }^{4}$. Toutes deux ajoutent que la spiritualité s'avère une ressource essentielle pour l'individu soigné, afin de promouvoir sa guérison. Pour leur part, Lemieux, McCarthy, Miller et Stoll considèrent la spiritualité comme étant la recherche d'un sens profond à la vie et aux événements de la vie, de même que la recherche d'une énergie créatrice 5 . La spiritualité, ajoute Stoll, agit comme la motivation et l'engagement de la personne envers des valeurs telles que l'amour, l'espoir et la beauté. Enfin, d'autres

3. M. LEININGER, "Transcultural Spirituality. A Comparative Care and Health Focus ", dans M.S. ROACH, dir., Caring from the Heart. The Convergence of Caring and Spirituality, New York, Paulist Press, 1997, p. 99 118 ; C.L. MONTGOMERY, «The Spiritual Connection. Nurses' Perceptions of the Experience of Caring ", dans D.A. GaUT, dir., The Presence of Caring in Nursing, New York, National League for Nursing, 1992, p. 39-52 ; D.P. O"NEILL et E.K. KENNEY, "Spirituality and Chronic Illness ", Image. Journal of Nursing Scholarship, 30/3 (1998) p. 275-280 ; K.B. WRIGHT, "Professional, Ethical, and Legal Implications for Spiritual Care in Nursing ", Image. Journal of Nursing Scholarship, 30/1 (1998) p. 81-83 ; S.G. WRIGHT et J. SAYRE-ADAmS, Sacred Space. Right Relationship and Spirituality in Healthcare, New York, Churchill Livingstone, 2000.

4. J. MACRAE, "Nightingale's Spiritual Philosophy and its Significance for Modern Nursing ", Image. The Journal of Nursing Scholarship, 27/1 (1995) p. 8-10 ; J. WATSON, Nursing. Human Science and Human Care, New York, National League of Nursing, 1988 ; IDEM, Postmodern Nursing and Beyond, London, Churchill Livingstone, 1999.

5. L. LEMIEUX, "Spiritualité et soins infirmiers ", L'infirmière du Québec, 6/6 (1999) p. 31-33, 36-37 ; P. MCCARTHY, "The Spirituality of Caring: Transformation to a Participatory Consciousness ", dans ROACH, dir., Caring from the Heart, p. 119-134 ; M.A. MILlER, "Culture, Spirituality, and Women's Health ", Journal of Obstetric, Gynecologic, and Neonatal Nursing, 24/3 (1995) p. 257-263 ; R.I. STOLL, " The Essence of Spirituality », dans V.B. CARSON, dir., Spiritual Dimensions of Nursing Practice, Philadelphia, Saunders, 1989, p. 4-23. 
auteurs définissent plutôt ce concept comme la transcendance et le dépassement de soi ${ }^{6}$.

En sciences infirmières, la distinction entre religion et spiritualité apparaît comme étant importante. Est-ce pour se distancier du passé religieux de la profession, pour éviter de s'éloigner de l'accent scientifique actuel ou est-ce le signe d'une vision renouvelée du spirituel ? Quoi qu'il en soit, le concept de spiritualité, englobant celui de religion et ne faisant pas appel à une affiliation religieuse particulière, permet à l'infirmière une approche à la fois plus universelle et plus personnelle auprès des individus et des familles soignées.

\section{La pratique professionnelle de l'infirmière}

D'entrée de jeu, précisons que la pratique professionnelle de l'infirmière est centrée sur le soin à la personne ou à la famille qui vit des expériences de santé en interaction continue avec son environnement ${ }^{7}$.

Les aspects du soin infirmier les plus connus, parce que les plus visibles, sont du domaine de l'instrumental et de l'éducatif : l'infirmière prépare un soluté, distribue des médicaments ; elle surveille le moniteur cardiaque ou le moniteur fœtal; elle installe la lunette nasale ou le cathéter urinaire ; elle aide la nouvelle mère à positionner son bébé pour l'allaitement, elle lui parle des bienfaits du lait maternel, elle répond à des questions, elle réfère à des ressources, elle suggère de consulter le médecin... les exemples sont innombrables. Cependant, les aspects réflexifs et expressifs du soin infirmier sont moins connus, parce

6. J.E HaAse, T. Britt, D.D. Coward, N. Kline Leidy et P. E. Penn, "Simultaneous Concept Analysis of Spiritual Perspective, Hope, Acceptance and Transcendence ", Image. Journal of Nursing Scholarship, 24/2 (1992) p. 141-147 ; M. Newman, Health as Expanding Consciousness, New York, National League for Nursing Press, $1994^{2}$; P. G REED, « Toward a Nursing Theory of Self-Transcendence. Deductive Reformulation Using Developmental Theories ", Advances in Nursing Science, 13/4 (1991) p. $64-$ 77 ; idem, "An Emerging Paradigm for the Investigation of Spirituality in Nursing ", Research in Nursing and Health, 15 (1992) p. 349-357 ; M.S. ROACH, "Reflections of the Theme ", dans ROACH, dir., Caring from the Heart, p. 7-20.

7. S. Kérouac, J. Pepin, F. Ducharme, A. Duquette et F. Major, La pensée infirmière, Laval, Études Vivantes, 1994. 
que davantage invisibles. Des auteurs nous rappellent que la «pratique infirmière englobe la création d'un soin individualisé, l'utilisation de soi en tant qu'outil thérapeutique et l'intégration de savoir-faire spécifiques ${ }^{8}$. L'infirmière ne fait pas qu'accomplir des tâches, elle accompagne, elle soutient, elle renforce la personne ou la famille lors d'expériences de santé ou de transitions de vie. C'est à la fois par sa manière d'être, son engagement, sa présence vraie et authentique qu'elle donne forme à son idéal moral de soin ${ }^{9}$ que ce soit dans son accompagnement à la phase intense du travail à la naissance, lors de l'annonce d'un diagnostic, lors d'une crise de dyspnée, au moment de la décision d'héberger ou non un parent âgé, ou à la mort d'un être cher.

En soignant, les infirmières sont constamment interpellées par des expériences humaines qui posent des questions fondamentales comme celles du sens de la vie, de la santé, de la souffrance et de la mort. Ces questions se posent pour les personnes qu'elles soignent mais aussi pour elles-mêmes. Comment jour après jour faire face à la douleur, à l'inévitable, à l'incompréhension et demeurer toujours engagée, présente, prête à l'écoute ? Comment ne pas penser à sa propre vulnérabilité, à sa propre mort ? C'est essentiellement la quête de sens que suscitent les diverses expériences de santé, dans lesquelles nous englobons la maladie, qui ramène des infirmières à des valeurs fondamentales et qui en invite d'autres à abandonner l'opposition entre science et religion, science et spiritualité.

Dans leur quête de sens, les infirmières puisent des réponses à même leur pratique ; elles apprennent des personnes et des familles qu'elles soignent, à travers leur cheminement dans leurs expériences de santé. Elles lisent aussi, en dehors des textes scientifiques, tout ce qui présente l'humain comme indivisible, tout ce qui cherche à donner sens. Elles s'ouvrent. Elles examinent leurs propres valeurs traditionnelles. Elles explorent. Les pratiques de soins non invasives qui se réclament de la vision globale de la personne sont particulièrement résonnantes pour les infirmières : le toucher thérapeutique en est un exemple. "Dans un monde de haute technologie coûteuse, on [l'infirmière] redécouvre la

8. KÉROUAC et al., La pensée infirmière, p. 74 ; WATSON, Nursing. Human Science; H.E. PEPLAU, Interpersonal Relations in Nursing. A Conceptual Frame of Reference for Psychodynamic Nursing, NewYork, G.P. Putnam's Sons, 1952. 9. WATSON, Nursing. Human Science; idem, Postmodern Nursing. 
valeur thérapeutique du toucher humain ${ }^{10}$, de la relation authentique, du temps en présence de l'autre. Les infirmières ont soif de ce type de relation vraie. D'ailleurs, la plupart d'entre elles ont choisi la profession infirmière pour en faire le centre de leurs activités.

\section{Les écrits conceptuels et théoriques}

Les infirmières trouvent également des réponses dans les fondements conceptuels et théoriques de leur discipline. Issue de la pratique soignante des femmes, la pratique infirmière a longtemps été exercée dans un cadre religieux qui intégrait la notion d'âme. En 1860, la britannique Florence Nightingale, pionnière du nursing moderne, reconnaissait que la discipline infirmière devait s'intéresser aux lois de la Nature et de Dieu, concernant la relation entre le corps de la personne soignée et son environnement. Elle définissait la spiritualité comme le sens d'une présence plus grande que l'humain (sa vision de Dieu) et une conscience d'être lié de l'intérieur à cette réalité plus grande. Elle soutenait également que la spiritualité, intrinsèque à l'humain, est une ressource essentielle qui comporte un potentiel de guérison ${ }^{11}$.

Le développement des sciences et de la médecine moderne correspond à la période de sécularisation et de désaveu du savoir des femmes sur les soins ${ }^{12}$. Cette période a fait ombre au spirituel en mettant l'accent d'abord sur le fonctionnement du corps, puis sur la relation entre le corps et le psychique. Au cours des années 1960, l'intérêt pour les besoins humains et le développement des personnes, incite Virginia Henderson ${ }^{13}$ à proposer à l'infirmière la mission sociale suivante : conserver ou rétablir l'indépendance de la personne de sorte qu'elle puisse satisfaire elle-même ses besoins fondamentaux (respirer, boire et manger... ), dont le besoin d'agir selon ses croyances spirituelles. Ce

10. M. Giasson, G. LerouX, H. TARdif et L. Bouchard, « Le toucher thérapeutique : une nouvelle façon d'aider les personnes atteintes de démence de type Alzheimer ", L'Infirmière du Québec, 6/6 (1999) p. 38-47 (ici p. 40). 11. MACRAE, "Nightingale's Spiritual Philosophy ».

12. M.F. COLLIÈRE, «Invisible Care and Invisible Women as Health CareProviders ", International Journal of Nursing Studies, 23/2 (1986) p. 95112 ; F. SAILlant, "La part des femmes dans les soins de santé ", Revue internationale d'action communautaire, 28/68 (1992) p. 95-106.

13. V. Henderson, Principes fondamentaux des soins infirmiers, Suisse, Karger Basel, 1969. 
besoin est fondamental, mentionne Henderson, puisqu'il contribue au maintien de la globalité de la personne. "Ce besoin correspond à la nécessité dans laquelle se trouve tout individu d'agir [selon] ses valeurs fondamentales, [valeurs] qui donnent un sens à sa vie et qui entretiennent un sentiment d'espoir ${ }^{14}{ }^{»}$.

Par ailleurs, à partir des années 1970, les travaux de Madeleine Leininger en sciences infirmières ont permis de relier culture et soin. Dans ce contexte, Leininger ne parlait pas de spiritualité mais de la religion, des croyances et de la philosophie de vie, comme étant des facteurs qui influencent la santé et les soins de la personne, famille, ou communauté15. En 1997, Leininger discute spécifiquement de la notion de spiritualité, comme étant la connexion et la relation avec une force de la vie, un être suprême ou surnaturel comportant des références symboliques, qui offrent une signification et guident les croyances, espoirs et actions de la personne ${ }^{16}$. L'individu qui reçoit des soins infirmiers jugés incohérents avec ses croyances et ses valeurs présentera, précise cette théoricienne, des signes de conflits et de stress.

Également dans les années 1970, Martha Rogers présente une vision de la personne unitaire indivisible. Cette perspective, révolutionnaire à ce moment, donne naissance à un paradigme différent en sciences infirmières. Rogers décrit la personne et son environnement comme des champs d'énergie " pandimensionnels » (non linéaires) en constante interaction. Chacun (personne et environnement), considéré comme un tout indivisible, est caractérisé par le changement continuel et mutuel, qu'elle nomme "configuration " énergétique. L'infirmière, selon Rogers ${ }^{17}$, accompagne la personne dans la partici-

14. R. COSSETte, "La personne âgée et son besoin d'agir selon ses croyances spirituelles ", dans S. LAUZON et E. ADAM, dir., La personne âgée et ses besoins, St-Laurent, ERPI, 1996, p. 579-623 (ici p. 580).

15. M. LEININGER, "Leininger's Theory of Nursing. Cultural Care Diversity and Universality ", Nursing Sciences Quarterly, 1/4 (1988), p. 152160 ; IDEM, Transcultural Nursing. Concepts, Theories, Research and Practice, New York, McGraw Hill, $1995^{2}$.

16. LEININGER, "Transcultural Spirituality » (voir note 3).

17. M ROGERS, An Introduction to the Theoretical Basis of Nursing, Philadelphia, J. A. Davis, 1970 ; IDEM, " Rogers' Science of Unitary Human Being ", dans R.R. PARSE, dir., Nursing Science. Major Paradigms, Theories, and Critiques, Philadelphia, Saunders, 1987. 
pation délibérée de celle-ci au processus de changement. Bien que Rogers ne référait pas directement à la spiritualité, ses écrits comportaient des liens évidents avec ce concept, entre autres en ce qui concerne l'interaction entre la personne et son environnement ${ }^{18}$.

Inspirée par les travaux de Rogers, Rosemarie Parse présente une théorie de l'humain en devenir où la personne, un tout indivisible et unitaire, a une préoccupation de sens ${ }^{19}$. Selon cette théoricienne, l'infirmière accompagne la personne dans sa recherche de sens à l'expérience vécue. Ainsi, Parse suggère à l'infirmière d'harmoniser son rythme à celui de la personne de façon à l'aider à identifier ou à percevoir de nouvelles possibilités à travers cette expérience, qu'elle nomme co-transcendance, et ce dans le but de favoriser une qualité de vie.

Également inspirée par les travaux de Rogers, Margaret Newman présente une théorie de la santé comme étant l'expansion de la conscience ${ }^{20}$. En 1994, Newman précise que l'humain vise l'expansion de sa conscience à travers les différents processus de la vie. Elle explique que ces processus impliquent une transcendance du soi spatio-temporel vers le domaine spirituel. Ainsi, le rôle de l'infirmière consiste à accompagner la personne dans l'expansion de sa conscience, en aidant cette dernière dans ses interactions avec l'environnement. Pour ce faire, explique Newman, l'infirmière établit un partenariat qui vise à promouvoir non seulement la transcendance de soi de la personne soignée, mais aussi celle de l'infirmière.

En 1979, Jean Watson, toujours dans cet esprit d'humain unitaire, qu'elle nomme cette fois " corps-âme-esprit ", va beaucoup plus loin dans la reconnaissance de la dimension spirituelle de l'être. Pour cette auteure, l'âme s'avère être l'essence même de la personne, ce qui lui permet de transcender l'ici-maintenant vers une prise de conscience

18. D.S. MARTSOlF et J.R. MiCKLeY, "The Concept of Spirituality in Nursing Theories. Differing World-Views and Extent of Focus ", Journal of Advanced Nursing, 27 (1998) p. 294-303.

19. R.R. PARSE, Man-Living-Health. A Theory of Nursing, New York, John Wiley \& Sons, 1981 ; IDEM, "Human Becoming. Parse's Theory of Nursing ", Nursing Science Quarterly, 5 (1992) p. 35-42.

20. M. Newman, Health as Expanding Consciousness, New York, National League for Nursing Press, 1986. 
plus profonde sur le sens de la vie ${ }^{21}$. Le rôle de l'infirmière consiste alors à accompagner la personne dans sa quête de sens afin de promouvoir une harmonie corps-âme-esprit. S'inspirant d'une philosophie existentielle, phénoménologique et spirituelle, sa théorie du human caring 22 discute de l'engagement personnel, moral, spirituel et social de l'infirmière nécessaire au développement de la relation de caring transpersonnel entre la personne et l'infirmière. Cette relation de caring se veut tributaire de la qualité des soins, de même que des services infirmiers offerts à la population. Somme toute, le caring, considéré par Watson comme l'essence des soins infirmiers, contribue à promouvoir la dignité humaine, la guérison (healing) et l'expansion de la conscience de la personne soignée.

Ainsi, il appert qu'une vision unitaire de la personne a fait resurgir plus clairement la question du spirituel et du sens dans les conceptions $\mathrm{du}$ soin et de la discipline infirmière ${ }^{23}$. Cette vision unitaire a aussi contribué à la transformation des recherches et autres travaux de nature empirique en sciences infirmières.

\section{Les écrits empiriques}

En consultant les écrits empiriques, il s'avère possible de constater qu'un nombre croissant d'infirmières s'attardent à décrire des expériences de santé des personnes ou des familles sous l'angle des "significations » pour ceux qui vivent ces expériences. L'approche phénoménologique demeure une méthodologie souvent privilégiée dans ce contexte. Ainsi, plusieurs études ont contribué à l'avancement des connaissances en permettant de mieux comprendre le sens de l'expérience de la maladie chronique, de l'espoir, de la souffrance, de la mort, du deuil pour la personne et sa famille, ou encore du défi de la croissance à travers les déclins de la vieillesse pour ne nommer que quelques exemples. Ces études aident l'infirmière dans le renouvellement de sa pratique professionnelle. Les méthodologies de type qualitatif sont d'ailleurs réclamées en sciences infirmières, non seulement

21. WATSON, Nursing. Human Science.

22. WATSON, Nursing. Human Science; IDEM, Postmodern Nursing.

23. Newman, Health as Expanding Consciousness ; PARSE, Man-LivingHealth; ROGERS, An Introduction; WATSON, Nursing. The Philosophy. 
pour l'étude des expériences de santé mais aussi pour documenter le lien entre spiritualité et santé ${ }^{24}$.

Si nous avons identifié, en sciences infirmières, des écrits épars sur la spiritualité au cours des années 1980, il s'en trouve un nombre croissant au cours des années 1990. Ce nombre d'écrits de plus en plus important dans le domaine infirmier nous porte à croire que les discours sur le sens et la spiritualité ne sont pas de passage, ils ne sont pas une mode. Au contraire, ces écrits reflètent un effort considérable pour clarifier la notion même de spiritualité et le rôle de l'infirmière face à cette dimension essentielle de l'être humain. En effet, au cours d'une étude visant à identifier l'objet des analyses conceptuelles publiées entre 1976 et 1996 en sciences infirmières, Lauzon et Pepin ont découvert cinq analyses, toutes publiées entre 1992 et 1996, portant sur des concepts parents de la spiritualité 25 Trois analyses portaient sur la "spiritualité ", une portait sur la " perspective spirituelle » qui était distinguée des concepts parents " espoir ", " acceptation » et " transcendance ", et une dernière portait sur les « relations spirituelles».

Outre les analyses conceptuelles, les infirmières cherchent également à identifier les effets bénéfiques de la spiritualité, à décrire la détresse spirituelle vécue à travers diverses expériences de santé, à évaluer l'importance de la spiritualité pour ces personnes, à identifier les besoins spirituels des personnes et des familles, de même qu'à clarifier les buts poursuivis par un accompagnement infirmier.

En effet, plusieurs auteurs ${ }^{26}$; à partir d'observations cliniques ou d'études formelles, ont soulevé la question des effets bénéfiques de la

24. P. Boston, B.M. Mount, S. Orenstein et O. Freedman, "Spirituality, Religion, and Health. The Need for Qualitative Research ", Annales CRMCC, 34/6 (2001) p. 368-374.

25. S. LAUZON et J. PEPIN, « L'appréhension de la substance infirmière par l'examen de concepts ayant fait l'objet d'analyses ", Recherche en soins infirmiers, 63 (2000) p. 10-18.

26. V.B. CARSON, Spiritual Dimensions of Nursing Practice, Philadelphia, W.B. Saunders, 1989 ; B.M. DosseY, "The Psychophysiology of Bodymind Healing ", dans B.M. Dossey, L. Keegan, C.E. GuZZetTa et L.G. KolKMEIER, dir., Holistic Nursing. A Handbook for Practice, Maryland, An Aspen Publication, $1995^{2}$, p. 87-111 ; B.M. Dossey, C.E. GunZETTA, « Holistic Nursing Practice », dans DosseY et al., Holistic Nursing, p. 5-36; 
spiritualité sur la santé de la personne et de sa famille. Par exemple, dans une étude qualitative réalisée auprès d'une clientèle atteinte du SIDA à un stade avancé, Hall conclut que la spiritualité favorise l'attribution d'une signification à une maladie incurable, procurant ainsi un soulagement de la souffrance et de l'anxiété. Maddox constate également un accroissement du bien-être et de l'harmonie chez des personnes âgées malades, face au soutien et à l'espoir que procure la spiritualité pour supporter la souffrance.

Par ailleurs, à la lumière de quelques écrits ${ }^{27}$, il est possible de regrouper certains indicateurs de détresse spirituelle. Outre le changement de comportement discuté par Carson (hostilité, pleurs, dépression, isolement, apathie...), il s'avère possible d'identifier chez la personne soignée et les membres de sa famille une verbalisation de conflits internes relatifs à sa propre existence (ex., culpabilité face à la maladie chronique transmise génétiquement à un enfant...). L'écoute de ces expressions de détresse invite l'infirmière à prodiguer des soins qui tiennent compte de la dimension spirituelle. On peut également noter un questionnement quant aux implications morales d'un traitement proposé, une incertitude quant au sens de la vie et de la mort, de même qu'une colère envers Dieu. Une demande de voir un pasteur ou un agent de pastorale est toujours accueillie ${ }^{28}$.

De plus, certains auteurs ${ }^{29}$ ont suggéré à l'infirmière d'explorer systématiquement la spiritualité de la personne soignée et de sa

B.A HALL, "Patterns of Spirituality in Persons with Advanced HIV Disease ", Research in Nursing and Health, 21 (1998) p. 143-153 ; LEININGER, "Transcultural Spirituality »; LEMIEUX, "Spiritualité et soins infirmiers "; M. MADDOX, "Spiritual Wellness in Older Women ", Journal of Christian Nursing, 17/1 (2000) p. 27-29 ; C.A. MCCulloch, « How Spirituality Transforms the Care of Older Persons ", dans ROACH, dir., Caring from the Heart, p. 135-148 ; O’NeILl ET KeNNEY, "Spirituality and Chronic Illness »; Wright et SAYRE-ADAMS, Sacred Space.

27. CARson, Spiritual Dimensions ; B.M. Dossey, " Dynamics of Healing and the Transpersonal Self ", dans DOSSEY et al., Holistic Nursing, p. 39-57; LEMIEUX, "Spiritualité et soins infirmiers »; STOLL, "The Essence of Spirituality ».

28. LeMIEUX, "Spiritualité et soins infirmiers ».

29. Carson, Spiritual Dimensions; Dossey et GunZETTA, "Holistic Nursing Practice » ; LEMIEUX, "Spiritualité et soins infirmiers »; O’NEILL et 
famille. Par exemple, à partir des résultats de l'analyse conceptuelle de Burkhardt, Dossey et Guzetta ont élaboré un outil visant à appréhender la spiritualité à trois niveaux: signification et but, forces intérieures et enfin, interconnections ${ }^{30}$. Voici quelques questions suggérées dans cet outil : Qu'est-ce qui donne un sens à votre vie? Est-ce que votre maladie interfère avec vos buts dans la vie? Quels sont les choix disponibles pouvant promouvoir votre guérison? Quelles sont vos croyances? Est-ce que la confiancelfoi est importante dans votre vie? Est-ce que la confiancelfoi joue un rôle pour retrouver la santé ? Que faites-vous pour guérir votre âme? Utilisez-vous la relaxation, l'imagerie, la méditation, la prière? (traduction libre)

Une telle « évaluation » de la spiritualité permet d'ailleurs, comme l'affirment Lemieux et Sherwood, de discerner des besoins d'ordre spirituel, chez l'individu soigné et sa famille ${ }^{31}$. La personne cherche-t-elle à trouver la signification et le sens de son existence, à affirmer sa croyance en des valeurs suprêmes, à conserver l'espoir et la confiance vis-à-vis d'un futur incertain ou encore à s'accepter plus pleinement ? L'infirmière peut accompagner la personne et sa famille dans l'identification de ses besoins. Une fois les besoins identifiés, O’Neill et Kenney ${ }^{32}$ recommandent de clarifier les buts poursuivis par l'accompagnement infirmier. Ainsi les soins infirmiers peuvent contribuer à promouvoir l'intégrité et la dignité de la personne, à promouvoir les relations interpersonnelles (avec soi, les autres et un Autre suprême), de même qu'à respecter et faciliter la quête de sens entreprise par la personne ${ }^{33}$.

Enfin, plusieurs écrits ${ }^{34}$ mettent en évidence des interventions infirmières visant l'écoute des besoins spirituels de la personne soignée et de

KENNEY, "Spirituality and Chronic Illness »; G. SHERWOOD, « Developing Spiritual Care. The Search for Self », dans ROACH, dir., Caring from the Heart, p. 196-211.

30. M. BurKhARDT, "Spirituality : An Analysis of the Concept ", Holistic Nursing Practice, 3/3 (1989) p. 69-77 ; DOSSEY et GUNZETTA, " Holistic Nursing Practice ».

31. LEMIEUX, "Spiritualité et soins infirmiers "; SHERWOOD, « Developing Spiritual Care ».

32. O’NeILl ET KeNNEY, "Spirituality and Chronic Illness »

33. O’NeILl ET KENNEY, "Spirituality and Chronic Illness », citant Munley.

34. CARSON, Spiritual Dimensions ; DOSSEY, "Dynamics of Healing "; idem, "The Psychophysiology ; S. HalldórSDótTIR, "Suffering- 
sa famille. Par exemple, l'infirmière, inspirée par une philosophie basée sur le caring, peut offrir à la personne la possibilité de verbaliser sa perspective, ses inquiétudes et ses besoins ${ }^{35}$. Elle peut encourager l'individu à comprendre ses conflits d'ordre moral ou spirituel. L'infirmière peut également créer et faciliter un environnement propice au ressourcement ou aux pratiques de rituels ${ }^{36}$. Enfin, en plus d'accompagner l'individu de par sa présence authentique, l'infirmière peut encourager l'implication de personnes importantes sur le plan spirituel afin de l'aider dans sa quête de sens et dans sa guérison " corps-âme-esprit " ${ }^{37}$.

En conclusion, le sens de la santé et de la guérison, de même que le lien avec la spiritualité évoqué par Nightingale, restent toujours à explorer. En effet, Nightingale soutenait assez justement, il y a de cela plus de cent ans :

On a tendance à croire que la médecine guérit. Rien n'est moins vrai ; (...). Ni la médecine, ni la chirurgie ne peuvent faire autre chose que d'enlever des obstacles; ni l'une, ni l'autre ne guérit; seule la Nature peut guérir. (...) Et ce que fait le nursing dans les deux cas c'est de placer le malade dans les meilleures conditions pour que la Nature agisse sur lui. ${ }^{38}$

Reflection-Caring ", International Journal for Human Caring, 3/2 (1999) p. 15-20 ; LemieuX, "Spiritualité et soins infirmiers "; LEININGER, "Transcultural Spirituality ; MADDOX, "Spiritual Wellness in Older Women "; MCCUlLOCH, "How Spirituality Transforms "; MONTGOMERY, "The Spiritual Connection "; A. OLDNALl, "A Critical Analysis of Nursing. Meeting the Spiritual Needs of Patients ", Journal of Advanced Nursing, 23 (1996) p. 138-144 ; O’NEILl ET KENNEY, "Spirituality and Chronic Illness »; SHERWOOD, "Developing Spiritual Care »; I. TuCK, L. PULlEN et C. LYNN, "Spiritual Interventions Provided by Mental Health Nurses », Western Journal of Nursing Research, 19/3 (1997) p. 351-363 ; WATSON, Nursing. Human Science ; idem, Postmodern Nursing ; idem, "Human Caring and Suffering. A Subjective Model for Health Sciences ", dans R. TAYLOR et J. WATSON, dir., They shall not Hurt. Human Suffering and Human Caring, Boulder, CO, Colorado Associated University Press, 1989, p. 125-135 ; WRIGHT et SAYREADAMS, Sacred Space.

35. WATSON, Nursing. Human Science.

36. LEMIEUX, "Spiritualité et soins infirmiers ».

37. DOSSEY, "Dynamics of Healing ».

38. F. Nightingale, Notes on Nursing: What it is and what it is not, New York, Dover, 1969 (1860), p. 74-75. 
Il est certain que les mandats de la médecine, de la chirurgie et des sciences infirmières ont été depuis, revus et reformulés selon une vision plus contemporaine. Cependant, l'idée que la Nature, avec un grand " $\mathrm{N}$ », a un rôle à jouer en juxtaposition avec les fruits de la science est à considérer sérieusement. Des infirmières, et un nombre croissant d'autres professionnels de la santé, s'y attardent.

Le paradigme de l'holisme en même temps qu'une ouverture aux philosophies sous-jacentes aux approches complémentaires de soins contribuent à offrir aux infirmières les moyens qu'elles cherchaient pour faire face aux questions de sens qui émergent de leur pratique. Une telle croissance personnelle et professionnelle ne peut être atteinte, mentionne Watson ${ }^{39}$, que lorsque l'infirmière accepte d'être touchée elle même dans son " corps-âme-esprit ", par la souffrance et l'expérience de la personne soignée. De ce fait, soigner la personne entière " corps âme-esprit » redevient un aspect essentiel du rôle de l'infirmière, quel que soit son secteur d'activité ou la clientèle. Les discours sur le sens et la spiritualité, en sciences infirmières du moins, correspondent ainsi au retour aux sources de la signification du soin à la personne entière qui vit des expériences de santé dans son environnement.

\section{RÉSUMÉ}

Les infirmières accompagnent les personnes et les familles pour diverses transitions de la vie: la naissance, la maladie, la mort... Elles sont interpellées par ces expériences humaines qui posent des questions fondamentales sur le sens de la vie, de la santé, de la souffrance et de la mort. Issue de la pratique soignante des femmes, la pratique infirmière a longtemps été exercée dans un cadre religieux qui intégrait la notion d'âme. En 1860, la britannique Florence Nightingale, pionnière du nursing moderne, reconnaissait que la discipline infirmière devait s'intéresser aux lois de la Nature et de Dieu concernant la relation entre le corps et son environnement. Le développement des sciences et de la médecine moderne, période de sécularisation et de désaveu du savoir des femmes sur les soins, a fait ombre au spirituel en mettant l'accent d'abord sur le corps, puis sur la relation entre le corps et le psychique. Or, dans les années 1970, la vision de la personne unitaire indivisible (corps-âme-esprit) proposée par Martha Rogers (1970) and Jean Watson (1979) a fait resurgir en sciences infirmières la question du spirituel et offre, de concert avec les philosophies sous-

39. WATSON, « Human Caring ». 
jacentes aux approches complémentaires, des moyens pour faire face aux questions de sens qui émergent de la pratique. D'où deux préoccupations nouvelles, en ce qui a trait aux sources du spirituel et à la signification du soin donnée à la personne qui vit des expériences de santé dans son environnement.

\section{ABSTRACT}

Throughout history, nurses have always cared for people and families during various life transitions, such as birth, disease, and death. These human experiences have challenged nurses on the meaning of life, health, suffering, and death. Originally rooted in women-caring practices, nursing took place within a religious context that integrated the notion of a soul. In 1860, for example, Florence Nightingale, the pioneer of modern nursing, maintained that the nursing discipline is concerned with the laws of Nature and God in order to elucidate the body-environment relationships. However, the development of sciences, and particularly the development of modern medicine, began a period of secularism and rejection of women's knowledge. in which the spiritual perspective has been overshadowed. The focus of care then became the body and the relationship between body and psyche. In the 1970s, the vision of the person as unitary (body — spirit - mind) presented by Martha Rogers (1970) and Jean Watson (1979) was a re-emergence of the spiritual dimension within the discipline. This unitary vision of the person, as well as the existential philosophies underlying the complementary caring approaches, offer nurses the means to address the questions pertaining to "meaning" which emerges in their practice. This renewed discourse is a return to the sources of the spiritual and the preoccupation of the meaning of caring in the human health experience. 